\title{
Dynamics of a two-level atom in a double perpendicular cavity
}

\author{
Julio C. García-Melgarejo ${ }^{* 1}$, Néstor Lozano-Crisóstomo ${ }^{1}$, Ponciano Rodríguez-Montero ${ }^{1}$, \\ and J. Javier Sánchez-Mondragón ${ }^{1}$ \\ ${ }^{l}$ Departamento de Óptica, Instituto Nacional de Astrofisica, Óptica y Electrónica, Luis Enrique Erro 1, 72840, \\ México
}

Received February 27, 2015; accepted April 13, 2015; published June 30, 2015

\begin{abstract}
We propose a variation of the Jaynes-Cummings Model (JCM), which consists of a Two-Level Atom (TLA) within a cross cavity configuration. This new geometry is a suitable tool to understand the origin of new QED features that the original JCM does not reveal, showing revivals in cases where they are not expected to appear.
\end{abstract}

The Jaynes-Cummings Model (JCM) describes the interaction between matter and a fully quantized field in a perfect one-dimensional cavity. The JCM has been the workhorse of Cavity Quantum Electrodynamics (CQED) for a number of decades [1]. Many authors have investigated several JCM generalizations to take into account more complex physical processes but not new QED features. The original one-dimensional arrangement has been studied in different contexts with interesting contributions [2-5]. Its interaction with more QED fields brought a wide variety of new cases, more complex atomic systems and more realistic experimental descriptions have given a thorough analysis of the model. That includes N-TLA and multimode cavities, in particular the driven and the two-mode JCM. However, they have not introduced a new distinctive QED feature. The JCM has been under continuous study for the last decades and has proved to be an ideal testing ground of fundamental concepts because it is one of the few fully soluble models in quantum optics. Despite the model simplicity, it shows the QED cavity field complexity through atomic interaction. Since then, other essential QED behaviors have been unveiled in other quite simple systems, such as a beam splitter, that suggest that new geometries would unveil a new QED nature. That will be discussed elsewhere [6].

Our recent work has been on a variation of the original one-dimensional problem which, however, allowed for an additional quantum feature. It consists of a TLA, with a real atomic dipole $\mathbf{d}=\mathbf{d}_{r}\left(\sigma_{+}+\sigma_{-}\right)$, placed between two perpendicular cavities, oriented along the $y$ and $x$ directions and fields on the $x$ and $y$ directions, which we will refer as X-cavity for short. It is relevant to inquire on the effects that our proposal has on the original JCM dynamics, in particular, the second arm effect. For

*E-mail: j_melgarejo@inaoep.mx instance, we study the revivals and photon number dynamics, $n_{a}=a^{\dagger} a$ and $n_{b}=b^{\dagger} b$, on the X-cavity arms.

The field in this configuration is described by:

$$
\mathbf{E}=E_{x}\left(a+a^{\dagger}\right) \mathbf{e}_{x}+E_{y}\left(b+b^{\dagger}\right) \mathbf{e}_{y},
$$

where $E_{x}$ and $E_{y}$ are the fields propagating along the $y$ and $x$ axis respectively, both with linear polarizations described by the unit vectors $\mathbf{e}_{x}$ and $\mathbf{e}_{y}$, respectively. The fields operators $a$ and $b$ obey the standard commutation rules of Bosonic operators $\left[a, a^{\dagger}\right]=1$ and $\left[b, b^{\dagger}\right]=1$ and there is no interaction among them $[a, b]=0$.

The Hamiltonian that describes this physical system, under the Rotating Wave Approximation (RWA), is:

$$
\begin{aligned}
h(a, b)= & \frac{1}{2} \hbar \omega_{0} \sigma_{z}+\hbar \omega_{y} a^{\dagger} a+\hbar \omega_{x} b^{\dagger} b+ \\
& +\hbar g_{x}\left(a \sigma_{+}+\sigma_{-} a^{\dagger}\right)+\hbar g_{y}\left(b \sigma_{+}+\sigma_{-} b^{\dagger}\right) .
\end{aligned}
$$

Here $\omega_{0}$ is the atomic transition frequency while $\omega_{y}$ and $\omega_{x}$ denote the modes frequency along the $x$ and $y$ directions respectively. The respective TLA coupling strength with the fields in the $x$ and $y$ directions is given by $g_{x}$ and $g_{y}$ where $g_{i}=E_{i} \mathbf{e}_{i} \cdot \mathbf{d}_{r} / \hbar$ with $i=x, y$. Furthermore, the atomic operators are given by $\sigma_{+}=|e\rangle\left\langle g\left|, \quad \sigma_{-}=\right| g\right\rangle\langle e|$, and $\sigma_{z}=|e\rangle\langle e|-| g\rangle\langle g|$, where $|g\rangle$ denotes the ground state and $|e\rangle$ is the excited state.

It is convenient to formulate the problem in terms of the mean cavities frequency $\tilde{\omega}=\left(\omega_{x}+\omega_{y}\right) / 2$, the cavities asymmetry $\delta=\left(\omega_{x}-\omega_{y}\right) / 2$ and the atomic detuning $\Delta=\omega_{0}-\tilde{\omega}$ with respect to $\tilde{\omega}$. The overall strength of the atom-Xcavity fields can be parameterized in terms of the effective coupling $g_{\text {eff }}=\sqrt{ }\left(g_{x}{ }^{2}+g_{y}{ }^{2}\right)$ and a convenient dynamical parameterization is obtained from the ratio $E_{y} \mathbf{e}_{y} \cdot \mathbf{d}_{r} /\left(E_{x} \mathbf{e}_{x} \cdot \mathbf{d}_{r}\right)=\tan \theta / 2=g_{y} / g_{x}$ that defines the dynamical unitary transformation:

$$
\left(\begin{array}{l}
A \\
B
\end{array}\right)=\left(\begin{array}{cc}
\cos \frac{\theta}{2} & \sin \frac{\theta}{2} \\
-\sin \frac{\theta}{2} & \cos \frac{\theta}{2}
\end{array}\right)\left(\begin{array}{l}
a \\
b
\end{array}\right),
$$


in terms of the strength of the coupling between the TLA and the fields:

$$
\begin{aligned}
& \cos \frac{\theta}{2}=\frac{g_{x}}{g_{\text {eff }}}, \\
& \sin \frac{\theta}{2}=\frac{g_{y}}{g_{\text {eff }}} .
\end{aligned}
$$

The new field operators obey the commutation rules $\left[A, A^{\dagger}\right]=1, \quad\left[B, B^{\dagger}\right]=1 \quad$ and $[A, B]=0$. Moreover, this transformation leaves the total photons number $n_{T}=a^{\dagger} a+$ $b^{\dagger} b=A^{\dagger} A+B^{\dagger} B=n_{a}+n_{b}=n_{A}+n_{B}$ unchanged.

The transformation given in the Eq.(3) not only defines new field operators but also new states. In terms of the new field operators, and by assuming symmetric cavities $\delta=0$, the Hamiltonian acquires the mathematical structure of the JCM Hamiltonian:

$$
\begin{aligned}
H(A, B)= & \hbar \tilde{\omega}\left(\sigma_{+} \sigma_{-}+A^{\dagger} A\right) \\
& +\frac{1}{2} \hbar \Delta \sigma_{z}+\hbar g_{\text {eff }}\left(A \sigma_{+}+\sigma_{-} A^{\dagger}\right)+\hbar \tilde{\omega} B^{\dagger} B .
\end{aligned}
$$

Clearly, the Hamiltonian given in Eq. (5) resembles the dynamics of a TLA interacting with a pseudo mode of the field described by $A$ and that of a field $B$ that has no interaction with the system. The structure of the JCM allows to identify the following motion constants [7]:

$$
\begin{aligned}
& C_{A}=\frac{1}{2} \Delta \sigma_{z}+g_{\text {eff }}\left(A \sigma_{+}+\sigma_{-} A^{\dagger}\right), \\
& N_{A}=\sigma_{+} \sigma_{-}+A^{\dagger} A, \quad n_{B}=B^{\dagger} B .
\end{aligned}
$$

Let us consider the initial state of the field prepared in a particular and convenient state in order to point out the second cavity arm effects on the JCM, in particular in the strong field JCM limit. Indeed, the coherent state is the most classical QED state. However, its atomic inversion is not our most familiar semiclassical picture, as shown in Figs. 1 and 2 of [8], and its revivals and short time decay have become CQED landmarks [9]. In spite of that, the Fock state JCM atomic inversion still resembles our semiclassical expectations [10]:

$$
\begin{aligned}
\left\langle n, m\left|\sigma_{z}(t)\right| n, m\right\rangle & =1-\frac{2 \Omega_{k}^{2}(0)}{\Omega_{k}^{2}} \sin ^{2}\left(\frac{\Omega_{k}}{2} t\right), \\
\Omega_{k}^{2} & =4 g^{2}\left[n+\frac{1}{2}(m+1)\right]+\Delta^{2},
\end{aligned}
$$

and it will be our choice because of its pure oscillatory behavior. In Eq. (7), $n$ is the Fock state eigenvalue, $m= \pm 1$ are the $\sigma_{z}$ eigenvalues and $\Omega_{k}$ is the JCM Rabi frequency. More complex states include a photon distribution superposition. In particular, for a coherent state the on-resonance revival time is $T_{R}{ }^{C}=2 \pi \sqrt{ }(\tilde{n}) / g$, where $\tilde{n}$ is the average photon number. In the $\mathrm{X}$-cavity JCM, $\Omega_{k}$ depends on the effective coupling coefficient and the $A$ photon number but the photon distribution changes according to Eq. (3). Let us choose the horizontal cavity field in a $M$ photons Fock state and the vertical field prepared in a vacuum state; then the initial state, in the new representation, is given by:

$$
\begin{aligned}
|M, 0\rangle \rightarrow & \sum_{k=0}^{M}(-1)^{k} \sqrt{\frac{M !}{k !(M-k) !}} \\
& \left.\left(\cos \frac{\theta}{2}\right)^{M-k}\left(\sin \frac{\theta}{2}\right)^{k}|M-k, k\rangle\right\rangle,
\end{aligned}
$$

where the double ket denotes a Fock state in the new representation $\left.\left.A^{\dagger} A B^{\dagger} B|M, N\rangle\right\rangle=M N|M, N\rangle\right\rangle$. The effects of a binomial distribution field have been reported in the frame of the JCM [11] but not on this quantum interpretation. For the JCM limit, let's consider $\sin (\theta / 2) \rightarrow 0$ and we recover the Fock state in Eq. (8). Another interesting limit is the very large photon number while keeping the Rabi frequency constant. When the atomic dipole is weakly coupled to the horizontal cavity $g_{x}<<g_{y}$, in such a way that the quantity $\mu_{\theta}=M \cos ^{2}(\theta / 2)$ remains constant, then the atomic inversion is given by:

$$
\left\langle\sigma_{z}(t)\right\rangle \approx \sum_{k=0}^{\infty}\left[1-\frac{8 g_{\text {eff }}^{2}(k+1)}{\Omega_{k}^{2}} \sin ^{2}\left(\frac{\Omega_{k}}{2} t\right)\right] \frac{\mu_{\theta}^{k}}{k !} e^{-\mu_{\theta}}
$$

where the Rabi's frequency is given by $\Omega_{k}{ }^{2}=\Delta^{2}+4 g_{\text {eff }}^{2}(k+1)$ as in Eq. (7). It is well known that the Eq. (9) sum produces the collapses and revivals [8-9]. Beyond that limit, we show in Fig. (1) the on-resonance $(\Delta=0) \mathrm{X}$-cavity Atomic inversion collapses and revivals, for $M=25$ photons, in two specific cases $g_{x}=g_{y} \quad\left[g_{\text {eff }}\right.$ $\left.=\sqrt{ }(2) g_{x}\right]$ and $2 g_{x}=g_{y}\left[g_{e f f}=\sqrt{ }(5) g_{x}\right]$ in units of a coherent state revival time $T_{R}{ }^{C o h}=2 \pi \sqrt{ }\left(\tilde{n}_{T}\right) / g_{\text {eff }}$. The role of $g_{\text {eff }}$ is quite clear, both in the revivals and the short time decay, as in their binomial photon distribution tendency to a Poisson distribution.
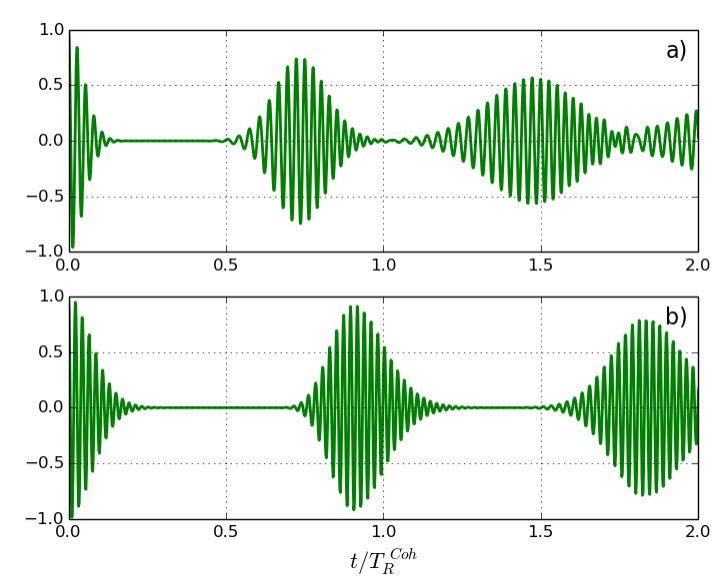

Fig. 1. X-cavity Atomic inversion collapses and revivals $\left\langle\sigma_{\mathrm{z}}(\mathrm{t})\right\rangle$, on resonance $(\Delta=0)$, for $M=25$ and a) $g_{x}=g_{y}\left[g_{\text {eff }}=\sqrt{ }(2) g_{x}\right]$ and b) $2 g_{x}=g_{y}$ $\left[g_{\text {eff }}=\sqrt{ }(5) g_{x}\right]$. 
In order to understand the revivals dynamics of a cross cavity JCM, one needs to know not only the time evolution of the atomic inversion but also the photon number in each arm $n_{a}=a^{\dagger} a$ and $n_{b}=b^{\dagger} b$. This coupled behavior of the cavities photons can be understood by writing the number operators $n_{a}$ and $n_{b}$ in terms of the new operators defined in the Eq. (3):

$$
\begin{aligned}
& a^{\dagger} a=\cos ^{2} \frac{\theta}{2} A^{\dagger} A-\sin \frac{\theta}{2} \cos \frac{\theta}{2}\left(A B^{\dagger}+B A^{\dagger}\right)+\sin ^{2} \frac{\theta}{2} B^{\dagger} B, \\
& b^{\dagger} b=\sin ^{2} \frac{\theta}{2} A^{\dagger} A+\sin \frac{\theta}{2} \cos \frac{\theta}{2}\left(A B^{\dagger}+B A^{\dagger}\right)+\cos ^{2} \frac{\theta}{2} B^{\dagger} B .
\end{aligned}
$$

The first term in Eq. (10) provides the revival distribution because $A^{\dagger} A$ is connected to the atomic dynamics through the constant $N_{A}$. The second term gives the coupling between the cavities in terms of the pseudo modes $A$ and $B$ and the last term is proportional to the constant $n_{B}$. It is quite interesting to notice the average photon number behavior in each arm, which reminds us of a mode coupled system and therein the distinctive revival periodicity, see Fig. 2. Such photon behaviour shows a QED dephasing decaying amplitude to a decoupling cavity photon number limit in each arm. However, we shall not expect a long term steady state due to the interference between the broadening revivals that define the asymptotic behavior [6].
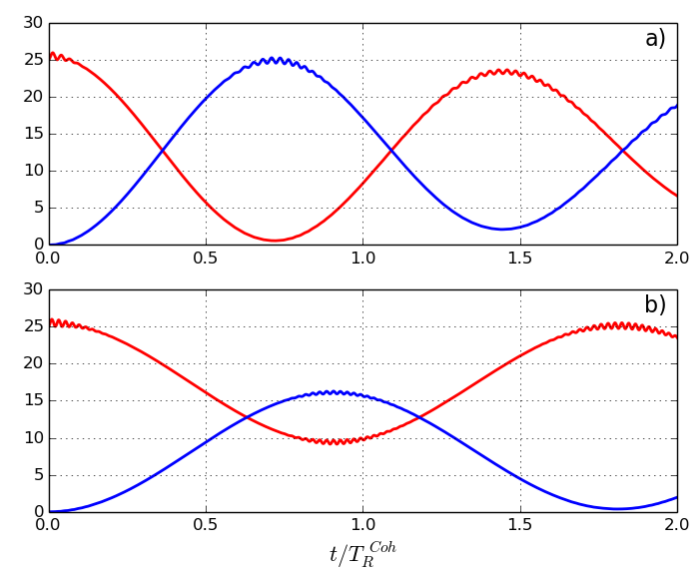

Fig. 2. X-cavity photon Coupling by the on-resonance TLA shown through the average of the photon number operators $n_{a}=a^{\dagger} a$ (red line) and $n_{b}=b^{\dagger} b$ (blue line). The system is prepared as in Fig. 1.

The addition of a second cavity is the origin of all those new QED features where collapses and revivals were obtained; even in those states where they are unexpected such as TLA in Fock state cavities. What is more, the TLA behaves as a coupler of their cavities modes with a QED dephasing decay and a revival period, therefore as a function of the photon number. The analogy with a dynamical quantum lossless beam splitter is shown in the large photon number limit.
We would like to dedicate this article to Profs. J. Mostowski, K. Rzazewski, A. Kujawski, I. BiałynickiBirula, J. H. Eberly and in the memory of K. Wódkiewicz. J.C. García-Melgarejo and N. Lozano-Crisóstomo express their appreciation to CONACyT for their graduate scholarships (235174 and 235214). CONACyT supported this work under contract CB-2008/101378.

\section{References}

[1] E.T. Jaynes, F.W. Cummings, Proc. IEEE 51, 1 (1963)

[2] M. Wilkens, P. Meystre, Phys. Rev. A 43, 7 (1991).

[3] I.V. Jyotsna, G.S. Agarwal, Opt. Commun. 99, 344 (1993).

[4] M.S. Abdalla, M. Abdel-Aty, A.-S.F. Obada, Opt. Comm. 211, 225 (2001).

[5] M.A. Marchiolli, R.J. Missori, J. Phys. A: Math. Gen. 36, 49 (2003).

[6] J.C. García-Melgarejo, Ph.D. Thesis. INAOE (2015).

[7] J.R. Ackerhalt, K. Rzazewski, Phys. Rev. A 12, 6 (1975)

[8] J.H. Eberly, N.B. Narozhny, J.J. Sanchez-Mondragon, Phys. Rev. Lett. 44, 20 (1980).

[9] N.B. Narozhny, J.J. Sanchez-Mondragon, J.H. Eberly, Phys. Rev. A 23, 1 (1985).

[10] L. Allen, J.H. Eberly, Optical resonance and two-level atoms (New York, Wiley 1975).

[11] A. Josi, R.R. Puri, J. Modern Optics 34, 11 (1987). 